\title{
Retinal Vessels Diameter Changes during Pregnancy in Type 1 Diabetes ${ }^{*}$
}

\author{
Aurore Mensah $^{1}$, Afrouz Ahmadzadeh ${ }^{1,2}$, Ann-Sofie Svanbom ${ }^{1,2}$, Lene Ringholm ${ }^{3}$, Birgit Sander ${ }^{1,2}$, \\ Henrik Lund-Andersen ${ }^{1,2}$, Elisabeth Mathiesen ${ }^{2,3}$, Michael Larsen ${ }^{1,2}$ \\ ${ }^{1}$ Department of Ophthalmology, Glostrup Hospital, Glostrup, Denmark; ${ }^{2}$ Faculty of Health Sciences, University of Copenhagen, \\ Copenhagen, Denmark; ${ }^{3}$ Department of Endocrinology, Rigshospitalet, Copenhagen, Denmark. \\ Email: retinaa@gmail.com
}

Received Februar 25 $5^{\text {th }}, 2013$; revised March 28 ${ }^{\text {th }}, 2013$; accepted April 30 ${ }^{\text {th }}, 2013$

Copyright (C) 2013 Aurore Mensah et al. This is an open access article distributed under the Creative Commons Attribution License, which permits unrestricted use, distribution, and reproduction in any medium, provided the original work is properly cited.

\begin{abstract}
Objective: Investigate how pregnancy-induced hormonal and hemodynamic changes affect retinal vessels caliber dynamic and the course of diabetic retinopathy. Methods: Retinopathy grading and vessel diameter measurement using digital fundus photographs in women with diabetes type 1 taken no more than one year before conception, during week 10 and/or 22, week 34, and within one year after delivery. Calibers were measured using a custom-developed semiautomated computer algorithm which identified the six largest arteries and six largest veins and calculated the central retinal artery equivalent (CRAE) and central vein equivalent (CRVE) diameters. Glycemic control was assessed quarterly by HbA1c. Systolic and diastolic blood pressure was assessed at week 10, 22 and 34. Results: Photographs at baseline, weeks 10, 22, 34 and after delivery were available in 28 patients, of whom 24 did not progress in retinopathy. For those whose level of retinopathy remained stable CRAE constricted from $138.80 \pm 21.27 \mu \mathrm{m}$ at baseline to $129.88 \pm$ $13.97 \mu \mathrm{m}$ at week $10(\mathrm{p}=0.0379) \mathrm{CRVE}$ constricted from $195.16 \pm 24.98 \mu \mathrm{m}$ at baseline to $185.97 \pm 21.96 \mu \mathrm{m}$ at week $10(\mathrm{p}=0.0400)$. The patients with retinopathy progression had a CRAE of $131.67 \pm 13.37 \mu \mathrm{m}$ at baseline and $132.08 \pm$ $11.07 \mu \mathrm{m}$ at week $10(\mathrm{p}=0.34)$ while CRVE was $195.33 \pm 24.92 \mu \mathrm{m}$ at baseline and $204.27 \pm 10.18 \mu \mathrm{m}$ at week $10(\mathrm{p}$ $=0.16$ ). Progressors had higher blood pressures at week 10. Conclusion: Retinal vessels constricted between prepregnancy and first trimester in women who did not experience diabetic retinopathy progression. The study suggests that the vessels constriction seen in early pregnancy contributes to reduce the risk of gestational microvascular complications and progression of diabetes in the eye.
\end{abstract}

Keywords: Pregnancy and Diabetes Type 1; Diabetic Retinopathy; Central Retinal Artery Equivalent; Central Retinal Vein Equivalent.

\section{Introduction}

Pregnancy is associated with increased short-term risk of development and progression of retinopathy in diabetic patients [1], an effect that may be related to pregnancy as such and the accompanying changes in circulation, blood pressure and hormone levels, or to some other factor, such as iatrogenic changes in glycemia directed at optimizing metabolic control and securing a favourable outcome of the pregnancy. Visual loss is associated with

\footnotetext{
*The authors have no proprietary or financial interest conflict to disclose.

Funding: No government or non-government grant as supported this study.

Financial disclosure: None of the authors involved in this study have financial disclosure or commercial interest related to this study.
}

microvascular lesions of the retina and these lesions are associated with retinal trunk vessel characteristics $[2,3]$. The physiological perturbations that occur provide an opportunity to observe dynamic changes in retinopathy and large retinal vessel. In this study, we assessed retinal artery and vein diameters before, during and after pregnancy in women with type 1 diabetes.

\section{Subjects and Methods}

This retrospective study included singleton pregnancies in women with pre-gestational type 1 diabetes referred to the Center for Pregnant Women with Diabetes at the Rigshospitalet in Copenhagen between January 2005 and December 2007. All patients had been treated with insu- 
lin from the onset of diabetes. The study included only women with planned pregnancies. All patients were invited to follow a glycemia and blood pressure optimization program in general practice or at the Steno Diabetes Center before they discontinued the use of anticonceptional strategies. They were referred to the Center for Pregnant Women with Diabetes no later than gestational week 14. All patients underwent fundus photographic retinopathy screening at the Rigshospitalet throughout the course of the study.

Inclusion required fundus photographs of acceptable quality from at least one eye at the following time points: prior to and no more than one year before conception, during gestational week 10 and/or 22, during gestational week 34, and after but no later than one year after delivery. Patients with pre-conceptional diabetic retinopathy levels higher than ETDRS 35 were excluded as were patients who had undergone laser retinal photocoagulation before pregnancy and patients with a history of eye disease other than diabetic retinopathy. Patients with uncontrolled systemic hypertension and patients using medications with vaso-active properties such as angiotensin receptor inhibitor before pregnancy or at the first trimester examination and women who were smoking were also excluded. The study adhered to the tenets of the Declaration of Helsinki. Because the study was entirely retrospective and did not involve biological material no ethics committee approval was required under Danish law.

Fundus photography, 20 and 50 degree digital grey scale red free and color at a nominal resolution of $1024 \times$ 1024 pixels (TRC-45, Topcon Corp., Tokyo, Japan) was made in both eyes after pupil dilation using phenyl epinephrine hydrochloride $10 \%$ and tropicamide $1 \%[4,5]$. Images were stored in an uncompressed format. For each session, the non-stereoscopic fundus photography protocol included images centered on the fovea, on the optic disc, on the ends of the temporal vascular arcades, and over the beginnings of the temporal vascular arcades. After exclusion of cases of inconsistent photographic quality 46 patients were available.

Diabetic retinopathy (DR) was graded according to an abbreviated version of the final Early Treatment Diabetic Retinopathy Study classification [6-8]. Baseline was defined as the pre-gestational fundus photography session. Severity was classified level 10 (no DR), levels 20 to 53 (non proliferative DR), and levels 61 to 85 , (proliferative DR). Change in retinopathy severity was assessed by side-by-side grading of pre-gestational fundus photographs with photographs made at gestational weeks 10 and 22, counting the gestational session that deviated most from the pre-gestational baseline. Diabetic macular edema was not assessed.

Retinal vessels calibers were measured using a custom-developed semi-automated computer algorithm [5].
Absolute dimensions were calculated assuming a vertical optic nerve head diameter of $1500 \mu \mathrm{m}$. A grid was placed over the 50 degrees digital image. Retinal vessels crossing the circular zone from 0.5 to 1.0 disc diameters from the margin of the optic nerve were analyzed. If bifurcations or branchings were found within the annular belt, the trunk was preferred to its branches unless the trunk segment was shorter than $80 \mu \mathrm{m}$. The program identified the six largest arteries and six largest veins and calculated the central retinal artery equivalent (CRAE) and central vein equivalent (CRVE) diameters according to empirical branching rules [9]. For the same eye, the same six arteries and veins were analyzed throughout the period of observation. Eyes were considered non-gradeable if one of the six largest arteries or veins could not be measured or if the image was deemed by the grader to be of poor quality compared to a standard image of acceptable quality and contrast.

Descriptive parameters included age at baseline, diabetes duration, and body-mass index (BMI) defined as weight divided by squared height. Glycemic control was assessed based on routine quarterly determination of $\mathrm{HbA}_{1 \mathrm{c}}$. Systolic and diastolic blood pressures were recorded at the Rigshospitalet at gestational weeks 10, 22 and 34 . Hypertension was defined as systolic blood pressure $\geq 135$ and or diastolic blood pressure $\geq 85 \mathrm{mmHg}$.

Statistical analyses were performed using commercial software (SAS 9.2 software for Windows; SAS institute, Inc., Cary, NC). Data are given as mean $\pm \mathrm{SD}$, median (Interquartile range) or fractions (\%). Categorical variables were compared by standard t-test.

One-way analysis of variance and summary change within subjects were analyzed using pair-wise tests. Vessel diameters are reported for only the right eye because CRAE and CRVE were correlated between eyes. Retinopathy is reported for both eyes. The level of statistical significance was set to $\mathrm{p}<0.05$.

\section{Results}

The study included 46 women with type 1 diabetes with a mean age of 31 (range 23 - 40) years. The mean duration of diabetes was 16 (range 2 - 29) years and mean BMI was 25 (range $19-32$ ) $\mathrm{kg} / \mathrm{m}^{2}$. Diabetic retinopathy in at least one eye was seen before gestation in 20 women $(43 \%)$, of whom 14 had level 20 retinopathy in both eyes, 4 had level 20 in one eye and 35 in the fellow eye while 2 had level 35 retinopathy in both eyes. In patients who had diabetic retinopathy at baseline progression of at least 2 levels (range 47 to 71 ) had occurred by the end of the second trimester in 4 women, who all had level 35 retinopathy in at least one eye at baseline, while retinopathy remained stable in 14 patients and regressed from level 35 to level 20 in 2 patients. In the 26 patients with- 
out retinopathy at baseline no development of retinopathy was seen.

In the 42 women in whom retinopathy did not develop or progress, pre-gestational $\mathrm{HbA}_{1 \mathrm{c}}$ was 7.28 (SD 1.02) \% and gestational week $10 \mathrm{HbA}_{1 \mathrm{c}}$ was 6.57 (SD 1.00) \% (Table 1, $\mathrm{p}<0.0001$ ) whereas the corresponding values for women who progressed were 7.85 (SD 1.45) \% and $6.98(1.24) \%(p=0.0354)$. Women who progressed to higher retinopathy levels had nominally higher blood pressures at week 10 than the remaining patients (Table 1) and were placed on intensified antihypertensive therapy.

In the 42 women in whom retinopathy did not develop or progress, CRAE was $131.95 \pm 18.53 \mu \mathrm{m}$ at baseline and $132.11 \pm 20.43 \mu \mathrm{m}$ after pregnancy $(\mathrm{p}=0.46)$ while the respective values for CRVE were $191.91 \pm 27.95 \mu \mathrm{m}$ and $194.80 \pm 35.05 \mu \mathrm{m}(\mathrm{p}=0.22)$ whereas the corresponding values for women who progressed in retinopathy severity, of whom 2 underwent photocoagulation therapy for proliferative diabetic retinopathy between weeks 20 and 34, CRAE was $131.67 \pm 10.29 \mu \mathrm{m}$ at baseline and $123.25 \pm 4.26 \mu \mathrm{m}$ after pregnancy $(\mathrm{p}=0.13)$, while the respective values for CRVE were $195.33 \pm 21.86 \mu \mathrm{m}$ and $186.44 \pm 38.74 \mu \mathrm{m}$ after pregnancy $(\mathrm{p}=0.35)$, corresponding to nominal arterial and venous diameter constrictions $6 \%$ and $5 \%$ in women who progressed.

A full series of photographs recorded at baseline, week $10,22,34$ and after delivery was available in 28 patients, of whom 24 did not experience any retinopathy progres- sion during pregnancy while 4 patients progressed 2 or more ETDRS levels, leading to photocoagulation for proliferative diabetic retinopathy in 2 of these patients between weeks 22 and 34.

In the 24 non-progressing patients the artery and vein diameters constricted during pregnancy, CRAE from $138.80 \pm 21.27 \mu \mathrm{m}$ at baseline to $129.88 \pm 13.97 \mu \mathrm{m}$ at week $10(\mathrm{p}=0.0379)$ and CRVE from $195.16 \pm 24.98$ $\mu \mathrm{m}$ at baseline to $185.97 \pm 21.96 \mu \mathrm{m}$ at week $10(\mathrm{p}=$ 0.0400 ; Table 2, Figure 1(a)), a reduction by $7 \%$ in both arteries and veins.

In the 4 patients who progressed no significant change was seen, CRAE being $131.67 \pm 13.37 \mu \mathrm{m}$ at baseline to $132.08 \pm 11.07 \mu \mathrm{m}$ at week $10(\mathrm{p}=0.34)$ and CRVE $195.33 \pm 24.92 \mu \mathrm{m}$ at baseline to $204.27 \pm 10.18 \mu \mathrm{m}$ at week 10 ( $\mathrm{p}=0.16$; Figure 1(b)), a nominal dilatation of $0.3 \%$ and $4.5 \%$, respectively. No other significant deviation from week 10 was seen in the non-progressing group during the rest of the period of pregnancy. In the progressing group, no other significant deviation from baseline was seen during the period of pregnancy, a nominal constriction that could be related to PRP was seen after delivery.

No significant correlation was found during the period of observation between CRAE/CRVE and age, $\mathrm{HbA}_{1 \mathrm{c}}$, blood pressure, BMI. Diabetes duration was on average 4 years longer and blood pressure was higher in subjects who experienced retinopathy progression during pregnancy than in patients who did not.

Table 1. Clinical characteristics in relation to diabetic retinopathy (DR) progression during pregnancy in women with type 1 diabetes.

\begin{tabular}{|c|c|c|c|c|c|c|}
\hline & \multicolumn{3}{|c|}{ No DR progression } & \multicolumn{3}{|c|}{ DR progression } \\
\hline & \multicolumn{3}{|c|}{$n=42$} & \multicolumn{3}{|c|}{$n=4$} \\
\hline & Mean & SD & $\mathbf{P}^{*}$ & Mean & SD & $\mathbf{P}^{*}$ \\
\hline Age & 31.22 & 3.98 & & 30.75 & 2.75 & \\
\hline $\begin{array}{c}\text { Diabetes duration } \\
\text { (years) }\end{array}$ & 16.14 & 7.19 & & 20.50 & 4.65 & \\
\hline BMI & 24.95 & 3.18 & & 25.43 & 4.40 & \\
\hline $\begin{array}{c}\text { Systolic }(\mathbf{m m H g}) \\
\text { Week } 10 \\
\text { Week } 22 \\
\text { Week } 34\end{array}$ & $\begin{array}{l}122.48 \\
122.39 \\
124.27\end{array}$ & $\begin{array}{c}11.82 \\
9.82 \\
11.93\end{array}$ & $\begin{array}{l}0.48 \\
0.21\end{array}$ & $\begin{array}{l}139.75 \\
131.75 \\
126.75\end{array}$ & $\begin{array}{l}8.66 \\
13.23 \\
11.41\end{array}$ & $\begin{array}{l}0.17 \\
\mathbf{0 . 0 5}\end{array}$ \\
\hline $\begin{array}{c}\text { Diastolic }(\mathbf{m m H g}) \\
\text { Week } 10 \\
\text { Week } 22 \\
\text { Week } 34\end{array}$ & $\begin{array}{l}72.20 \\
73.91 \\
76.95\end{array}$ & $\begin{array}{l}7.66 \\
6.91 \\
5.55\end{array}$ & $\begin{array}{c}0.10 \\
\mathbf{0 . 0 0 0 2}\end{array}$ & $\begin{array}{l}82.25 \\
82 \\
78.25\end{array}$ & $\begin{array}{l}2.63 \\
5.48 \\
5.32\end{array}$ & $\begin{array}{c}0.46 \\
0.0917\end{array}$ \\
\hline $\begin{array}{c}\text { HbA }_{1 \mathrm{c}}(\%) \\
\text { Before pregnancy } \\
\text { Week 10 } \\
\text { Week 22 } \\
\text { Week } 34\end{array}$ & $\begin{array}{l}7.28 \\
6.57 \\
5.92 \\
5.87\end{array}$ & $\begin{array}{l}1.02 \\
1.00 \\
0.43 \\
0.38\end{array}$ & $\begin{array}{l}<0.0001 \\
<0.0001 \\
<0.0001\end{array}$ & $\begin{array}{l}7.85 \\
6.98 \\
6.03 \\
5.90\end{array}$ & $\begin{array}{l}1.45 \\
1.24 \\
0.78 \\
0.47\end{array}$ & $\begin{array}{l}0.0354 \\
0.0145 \\
0.0217\end{array}$ \\
\hline
\end{tabular}

*Pair-wise comparison with week-10 value within each group, except for $\mathrm{HbA}_{1 \mathrm{c}}$, where comparison was with most recent pre-conceptional value. 
Table 2. Retinal vessels diameters in relation to retinopathy progression during pregnancy in women with type 1 diabetes.

\begin{tabular}{cccc}
\hline & $\begin{array}{c}\text { Before } \\
\text { pregnancy }\end{array}$ & $\begin{array}{c}\text { Gestational week } \\
\mathbf{1 0}\end{array}$ & $\begin{array}{c}\text { After } \\
\text { delivery }\end{array}$ \\
\hline $\mathrm{CRAE}$ & 138.80 & 129.88 & 135.22 \\
mean; $\mathrm{CI}_{95}[\mu \mathrm{m}]$ & $129.60 ; 148.0$ & $123.94 ; 135.92$ & $127.25 ; 143.19$ \\
$\mathrm{CRAE}$ change & & 8.91 & 3.58 \\
mean; $\mathrm{CI}_{95}[\mu \mathrm{m}]$ & & $0.54 ; 17.29$ & $-3.755 ; 10.926$ \\
$\mathrm{P}$ & & $\mathbf{0 . 0 3 7}$ & 0.32 \\
$\mathrm{CRVE}$ & 195.16 & 185.97 & 196.60 \\
mean; $\mathrm{CI}_{95}[\mu \mathrm{m}]$ & $184.35 ; 205.96$ & $176.48 ; 195.47$ & $181.61 ; 211.59$ \\
$\mathrm{CRVE}$ change & & 9.18 & -1.44 \\
mean; $\mathrm{CI}_{95}[\mu \mathrm{m}]$ & & $0.45 ; 17.909$ & $-10.22 ; 7.34$ \\
$\mathrm{P}$ & & $\mathbf{0 . 0 4 0}$ & 0.73 \\
\hline
\end{tabular}

$\mathrm{CI}_{95}$ Ninety-five per cent confidence interval; P Paired, t-test comparing data from week 10 and after delivery to values before pregnancy $(n=24)$.

\section{Discussion}

This exploratory study examined retinopathy and retinal vessel diameters before, during and after pregnancy in women with type 1 diabetes and found that women who experienced retinopathy progression were characterized by absence of retinal vessel contraction during pregnancy. Contraction of both retinal arteries and veins was seen in women without retinopathy progression during the first trimester. Women with retinopathy progression experienced a reduction in arterial blood pressure related to intensification of antihypertensive therapy between gestational weeks 10 and 22. Arterial blood pressure in women without retinopathy progression remained stable during this period and their antihypertensive therapy was not intensified. The 9 percent fraction of patients that progressed to higher levels of retinopathy is comparable to previous reports $[10,11]$.

The results of this study suggests that retinal vasodilatation during pregnancy is a risk factor for retinopathy progression. Furthermore, the study suggests that the potential interaction with antihypertensive therapy should be examined in more detail in a prospective study with extensive baseline characterization and a formal intervention protocol.

Our results are consistent with studies of men and non-pregnant women where large retinal vessel diameters have been found to be a risk factor for diabetic retinopathy [12]. Larger retinal vein diameters predicted 4 years progression of diabetic retinopathy and progression to proliferative diabetic retinopathy in the Wisconsin Epidemiologic Study of Diabetic Retinopathy [2]. Larger retinal artery diameters also predict incident retinopathy in adolescents [3]. In the present study, a nominal $7 \%$ constriction was observed in the first trimester in retinal arteries and veins in women without retinopathy progression. Women with progression demonstrated a nominal $4.5 \%$ dilatation of retinal veins at first trimester while artery diameters remained unchanged.

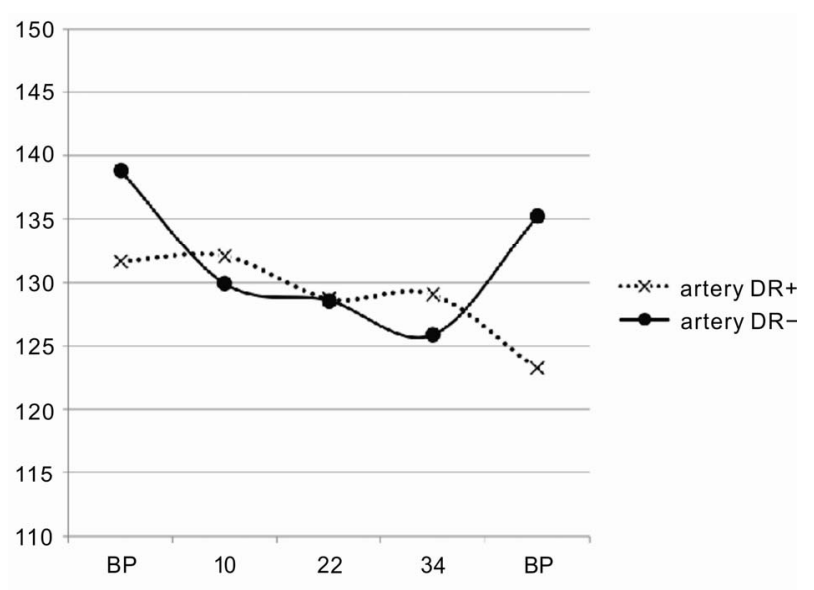

(a)

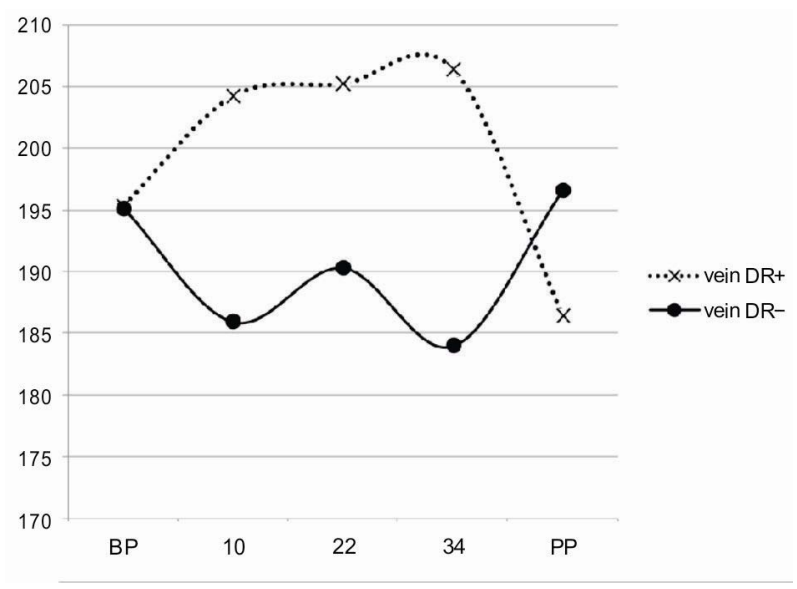

(b)

Figure 1. (a) Central retinal artery equivalent diameter in $\mu \mathrm{m}$ in pregnant women with type 1 diabetes who did not experience retinopathy progression during pregnancy (DR-, full line, $n=24$ ) and in women with diabetes who progressed to higher retinopathy levels $(\mathrm{DR}+$, dotted line, $n=4)$ in relation to gestational age (BP before pregnancy, numerical values representating, $P P$ post-partum); (b) Central retinal vein equivalent diameter in $\mu \mathrm{m}$ in pregnant women with type 1 diabetes who did not experience retinopathy progression during pregnancy $(\mathrm{DR}-$, full line, $n=24)$ and in women who progressed to higher retinopathy levels $(D R+$, dotted line, $n=4)$ in relation to gestational age (BP before pregnancy, numerical values representating, $P P$ post-partum).

Retinal vessel dilatation is associated with signs of endothelial dysfunction, in the form of an absent retinal vessel vasodilatory response to flicker-light stimulation [13]

Previous studies have demonstrated that retinal capillary blood flow is increased during pregnancy in women with diabetes compared to women without diabetes [14, 15], perhaps because hypoxia in the diabetic retina has led to exhaustion of retinal autoregulation $[16,17]$. Indeed larger retinal vein diameters have been shown in a 
population study to be associated with lower arterial oxygen saturation [18]. An experimental study of retinal volumetric blood flow during pregnancy in women with diabetes found an association between retinopathy progression and increasing retinal blood flow [19].

A previous study of retinal artery diameters in 45 pregnant women with diabetes found an association between smoking as well as smaller retinal artery diameters in the first trimester and lower rates of diabetic retinopathy progression [20]. It remains to be determined whether retinal vasodilation promotes retinopathy or retinopathy promotes vasodilation.

The present study did not find a relation between $\mathrm{HbA}_{1 \mathrm{c}}$ and vessel diameters or retinopathy, possibly because glycemia was systematically brought under control during a run-in period before conception. This may have reduced the risk of retinopathy progression during pregnancy, a phenomenon that may be induced by iatrogenic tightening of glycemia control rather than pregnancy itself [21-24].

The results of the present study suggest that retinal vessels contraction during early pregnancy is contributing to reduce gestational microvascular complications and progression of diabetic retinopathy. The study is limited by relatively small numbers and should be followed up by a larger prospective study.

\section{Acknowledgements}

The study was presented as an abstract at the Association for Research in Vision and Ophthalmology Conference 2010 in Fort Lauderdale, Florida, United States of America.

\section{REFERENCES}

[1] R. Axer-Siegel, M. Hod, A. Fink-Cohen, M. Kramer, D. Weinberger, B. Schindel, et al., "Diabetic Retinopathy during Pregnancy," Ophthalmology, Vol. 103, No. 11, 1996, pp. 1815-1819.

[2] R. Klein, B. E. Klein, S. E. Moss, et al., "Wisconsin Epidemiologic Study of Diabetic Retinopathy, the Relation of Vessel Caliber to the Incidence and Progression of Diabetic Retinopathy," Archives of Ophthalmology, Vol. 122, No. 1, 2004, pp. 76-83. doi:10.1001/archopht.122.1.76

[3] N. Cheung, S. L. Rogers, K. C. Donaghue, A. J. Jenkins, G. Tikellis and T. Y. Wong, "Retinal Arteriolar Dilatation Predicts Retinopathy in Adolescent with Type 1 Diabetes," Diabetes Care, Vol. 31, No. 9, 2008, pp. 1842-1846. doi: $10.2337 / \mathrm{dc} 08-0189$

[4] P. K. Kofoed, B. Sander, G. Zubieta-Calleja, L. Kessel and M. Larsen, "Retinal Vessel Diameters in Relation to Hematocrit Variation during Acclimatization of Highlanders to Sea Level Altitude," Investigative Ophthalmology \& Visual Science, Vol. 50, No. 8, 2009, pp. 39603963. doi:10.1167/iovs.08-3217
[5] N. C. Taarnhoj, M. Larsen, B. Sander, K. O. Kyvik, L. Kessel, J. L. Hougaard and T. I. Sorensen, "Heritability of Retinal Vessel Diameters and Blood Pressure: A Twin Study," Investigative Ophthalmology \& Visual Science, Vol. 47, No. 8, 2006, pp. 3539-3544. doi:10.1167/iovs.05-1372

[6] Early Treatment Diabetic Retinopathy Study Research Group, "Grading Diabetic Retinopathy from Stereoscopic Color Fundus Photographs an Extension of the Modified Airlie House Classification," Ophthalmology, Vol. 98, No. 5, 1991, pp. 786-806.

[7] Early Treatment Diabetic Retinopathy Study Research Group, "Fundus Photographic Risk Factors for Progression of Diabetic Retinopathy," Ophthalmology, Vol. 98, No. 5, 1991, pp. 823-833.

[8] C. P. Wilkinson, F. L. Ferris 3rd, R. E. Klein, P. P. Lee, C. D. Agardh, M. Davis, D. Dills, A. Kampik, R. Pararajasegaram, J. T. Verdaguer and Global Diabetic Retinopathy Project Group, "Proposed International Clinical Diabetic Retinopathy and Diabetic Macular Edema Disease Severity Scales," Ophthalmology, Vol. 110, No. 9, 2003, pp. 1677-1682.

[9] M. D. Knudson, K. E. Lee, L. D. Hubbard, T. Y. Wong, R. Klein and B. E. Klein, "Revised Formulas for Summarizing Retinal Vessel Diameters," Current Eye Research, Vol. 27, No. 3, 2003, pp. 143-149. doi:10.1076/ceyr.27.3.143.16049

[10] The Diabetes Control and Complications Trial Research Group, "Effect of Pregnancy in Microvascular Complications in the Diabetes Control and Complication Trial," Diabetes Care, Vol. 23, No. 8, 2000, pp. 1084-1091. doi:10.2337/diacare.23.8.1084

[11] M. Horvat, H. Maclean, L. Goldberg and G. W. Crock, "Diabetic Retinopathy in Pregnancy: A 12 Years Prospective Survey," British Journal of Ophthalmology, Vol. 64, No. 6, 1980, pp. 398-403.

[12] F. Islam, T. Nguyen, J. Wang, et al., "Quantitative Retinal Vascular Calibre Changes in Diabetes and Retinopathy: The Singapore Malay Eye Study," Eye, Vol. 23, No. 8, 2009, pp. 1719-1724. doi:10.1038/eye.2008.362

[13] A. Mandecka, J. Dawczynski, M. Blum, et al., "Influence of Flickering on the Retinal Vessels in Diabetic Patients," Diabetes Care, Vol. 32, No. 11, 2007, pp. 3048-3052. doi:10.2337/dc07-0927

[14] S. Loukovaara, R. Kaaja and I. Immonen, "Macular Capillary Blood Flow Velocity by Blue-Field Entoptoscopy in Diabetic and Healthy Women during Pregnancy and the Postpartum Period," Graefe's Archive for Clinical and Experimental Ophthalmology, Vol. 240, No. 12, 2002, pp. 977-982. doi:10.1007/s00417-002-0590-8

[15] S. Loukovaara, M. Harju, R. Kaaja and I. Immonen, "Retinal Capillary Blood Flow in Diabetic and Nondiabetic Women during Pregnancy and Postpartum Period," Investigative Ophthalmology \& Visual Science, Vol. 44, No. 4, 2003, pp. 1486-1491. doi:10.1167/iovs.02-0293

[16] E. Stefansson, M. D. Landers III and M. L. Wolbarsht, "Oxygenation and Vasodilatation in Relation to Diabetic and Other Proliferative Retinopathies," Ophthalmic Surgery, Vol. 14, No. 3, 1983, pp. 209-226. 
[17] A. Kifley, J. J. Wang, S. Cugati, T. Y. Wong and P. Mitchell, "Retinal Vascular Caliber, Diabetes and Retinopathy," American Journal of Ophthalmology, Vol. 143, No. 6, 2007, pp. 1024-1026. doi:10.1016/j.ajo.2007.01.034

[18] F. J. De Jong, M. W. Vernooij, M. K. Ikram, et al., "Arteriolar Oxygen Saturation, Cerebral Blood Flow and Retinal Vessel Diameters: The Rotterdam Study," Ophthalmology, Vol. 115, No. 5, 2008, pp. 887-892. doi:10.1016/i.ophtha.2007.06.036

[19] H. C. Chen, S. B. Newsom, V. Patel, J. Cassar, H. Mather and E. H. Kohner, "Retinal Blood Flow Changes during Pregnancy in Women with Diabetes," Investigative Ophthalmology \& Visual Science, Vol. 35, 1994, pp. 31993209.

[20] M. Larsen, L. B. Colmorn, M. Bonnelycke, R. Kaaja, I. Immonen, B. Sander and S. Loukovaara, "Retinal Artery and Vein Diameters during Pregnancy in Diabetic Women," Investigative Ophthalmology \& Visual Science, Vol. 46, No. 2, 2005, pp. 709-713. doi:10.1167/iovs.04-0604

[21] M. Vestgaard, L. Ringholm, C. S. Laugesen, K. L. Rasmussen, P. Damm and E. Mathiesen, "Pregnancy-Induced
Sight-Threatening Diabetic Retinopathy in Women with Type 1 Diabetes," Diabetic Medicine, Vol. 27, 2010, pp. 1-5. doi:10.1111/j.1464-5491.2010.02958.x

[22] The Diabetes Control and Complications Trial Research Group, "Retinopathy and Nephropathy in Patients with Type 1 Diabetes Four Years after a Trial of Intensive Therapy," The New England Journal of Medicine, Vol. 342, No. 6, 2000, pp. 381-389. doi:10.1056/NEJM200002103420603

[23] R. C. Temple, V. A. Aldridge, M. J. Sampson, R. H. Greenwood, P. J. Heyburn and A. Glenn, "Impact of Pregnancy on the Progression of Diabetic Retinopathy in Type 1 Diabetes," Diabetic Medicine, Vol. 18, No. 7, 2001, pp. 573-577. doi:10.1046/j.1464-5491.2001.00535.x

[24] G. T. Higgins, K. Khan and E. A. Pearce, "Glycemic Control and Control of Risk Factors in Diabetic Patients in an Ophthalmology Clinic: What Lessons Have We Learned from the UKPDS and DCCT Studies?" Acta Ophthalmologica Scandinavica, Vol. 85, No. 7, 2007, pp. 772-726. doi:10.1111/j.1600-0420.2007.00944.x 